\title{
Modification of electroluminescence and charge trapping in germanium implanted metal-oxide silicon light-emitting diodes with plasma treatment
}

\author{
A.N. Nazarov, ${ }^{2}$ W. Skorupa, ${ }^{1}$ Ja.N. Vovk, ${ }^{2}$ I.N. Osiyuk, ${ }^{2}$ A.S. Tkachenko, ${ }^{2}$ I.P. Tyagulskii, ${ }^{2}$ V.S. Lysenko, ${ }^{2}$ \\ T. Gebel, ${ }^{3}$ L. Rebohle, ${ }^{3}$ R.A. Yankov, ${ }^{3}$ T.M. Nazarova ${ }^{4}$ \\ 1 Institute of Ion Beam Physics and Materials Research, Forschungszentrum Rossendorf e.V., POB 510119, D-01314 \\ Dresden, Germany \\ 2 Institute of Semiconductor Physics, NAS of Ukraine, 45, prospekt Nauky, 03028 Kyiv, Ukraine \\ 3 Nanoparc GmbH, 45, Bautzner Landstrasse, D-01454 Dresden, Germany \\ 4 National Technical University “KPI”, 37, prospekt Peremogy, 03056 Kyiv, Ukraine
}

\begin{abstract}
We have studied the effect of plasma treatment on both the electroluminescent (EL) properties of Ge-implanted light-emitting metal-oxide silicon (MOS) devices and the charge trapping processes occurring therein. Under optimum conditions of plasma treatment, an appreciable increase in the device lifetime has been observed while maintaining the intensity of the light emission unchanged in the violet range of the spectrum. These phenomena are believed to be associated with recovery of the oxide network resulting from a relief of internal mechanical stresses and bond rearrangement that leads to a decrease in the generation efficiency of electron traps which are responsible for the device degradation.
\end{abstract}

Keywords: electroluminescence, nanoclusters, MOS structure.

Manuscript received 10.12.04; accepted for publication 18.05.05.

\section{Introduction}

The development of silicon-based light-emitting devices capable of operating at room temperature (RT) has recently gained in importance due to the need for efficient and inexpensive light sources, fabrication of which is fully compatible with the existing silicon device technology. One promising version of such a device consists of MOS structure in which the thermally-grown $\mathrm{SiO}_{2}$ film is ion-implanted with $\mathrm{Ge}$ [1-3] and is then processed by rapid thermal annealing (RTA) $[4,5]$. This MOS light-emitting device (MOSLED) typically uses a transparent layer of indium-tin oxide (ITO) on the top of the $\mathrm{SiO}_{2}$ film, and an $\mathrm{Al}$ layer on the back of the $\mathrm{Si}$ substrate as the current injecting electrodes [4]. A technologically important feature of a Ge ion-implanted and RTA-treated MOSLED is its ability to emit violet light (wavelength $\lambda=390 \mathrm{~nm}$ ) of an appreciably high intensity. Such MOSLEDs represent significant advancement towards combining optical and electronic processing on the same chip while achieving a maximal EL efficiency within the limits of the Si material properties.

Critical issues of the MOSLED performance are the device durability in terms of device operating lifetime, which has to be made sufficiently long, and the highfield injection-associated oxide degradation, which has to be prevented or at least retarded significantly in order to permit practical utilization [6-8].

The previous works [9-11] have shown that the radio-frequency (RF) plasma treatment (PT) of ionimplanted MOS structures may result in a considerable modification of their structural and electrical properties. In the present paper, we demonstrate that the optimized low-temperature RF treatment of MOSLEDs in hydrogen containing plasma is an efficient and straightforward way of improving their performance and high-field injection behavior. To the best of our knowledge, the effect of such a plasma treatment on the EL and charge trapping properties of high-dose Geimplanted MOSLEDs has not yet been reported.

\section{Experimental}

MOSLED structures were fabricated using an $n$-type (100) Si wafer on which a 200 nm-thick layer of thermal $\mathrm{SiO}_{2}$ was first grown. $\mathrm{Ge}^{+}$ions were subsequently implanted into the $\mathrm{SiO}_{2}$ layer at the energy of $100 \mathrm{keV}$ using the dose of $2.4 \cdot 10^{16} \mathrm{~cm}^{-2}$ to form a Ge depth profile with a peak centered in the middle of the oxide. Following implantation, the $\mathrm{SiO}_{2} / \mathrm{Si}$ structure was given an RTA at $1000^{\circ} \mathrm{C}$ for $6 \mathrm{~s}$ in a nitrogen ambient, taking into account the fact that such an annealing would change the Ge implant profile insignificantly [7, 8] and lead to the maximal EL intensity [12]. An Al layer was 


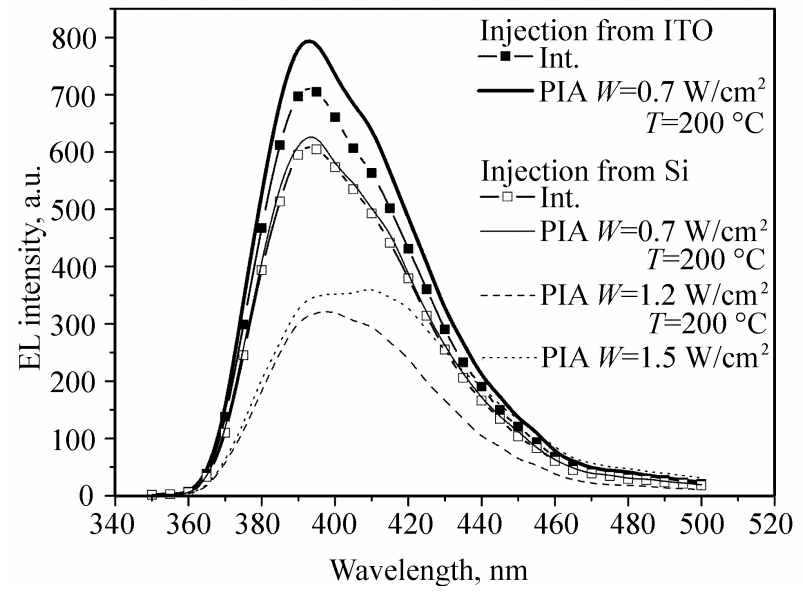

Fig. 1. EL spectra of Ge-implanted $\mathrm{SiO}_{2}$ before and after plasma treatment using different power densities.
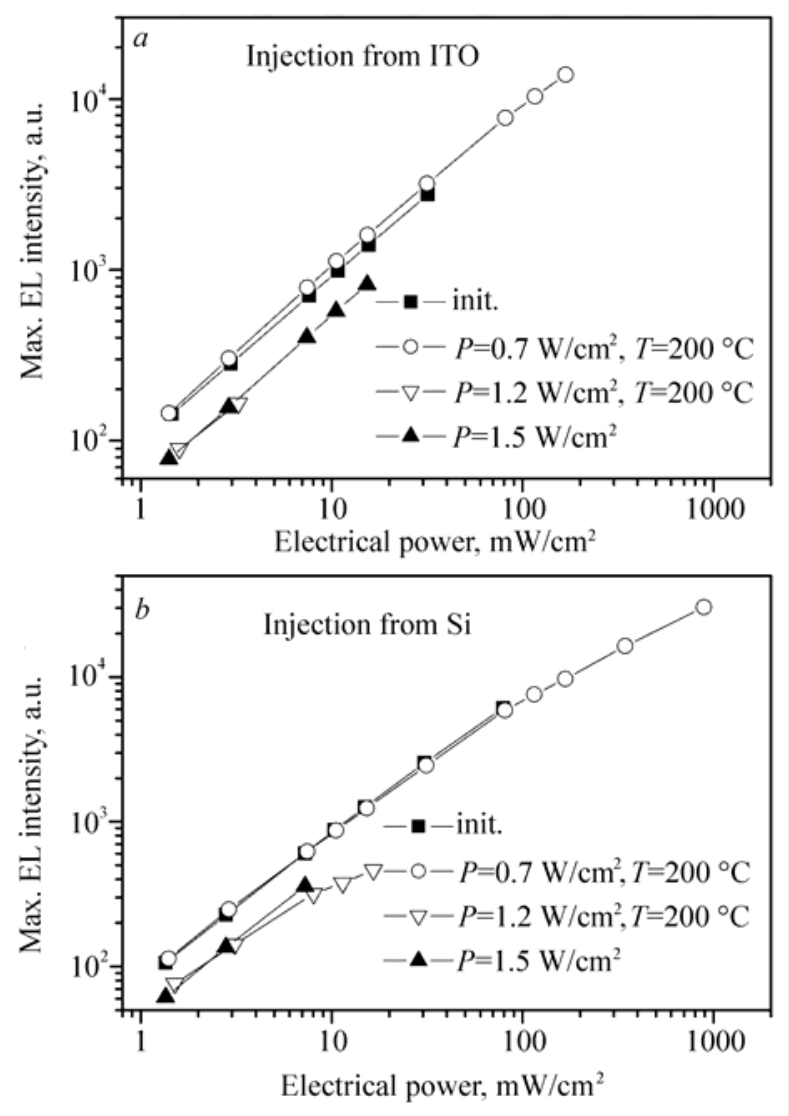

Fig. 2. Variation of the maximal EL intensity with electric input power measured at a wavelength of $390 \mathrm{~nm}$ prior to and following PT for electron injection from ITO electrode (a) and Si substrate (b).

deposited onto the back of the Si substrate, and an ITO electrode of $80 \mathrm{~nm}$ thickness was sputter-deposited onto the $\mathrm{SiO}_{2}$ layer to form a MOSLED structure.

The plasma treatment was finally performed on the MOSLED structure so fabricated using an RF (13.56 MHz) plasma generated in a low pressure, parallel plate (diode type) reactor, with the ITO electrode being exposed to the plasma discharge. The plasma operating gas was a mixture of $90 \%$ nitrogen and $10 \%$ hydrogen. The plasma generation power density was in the 0.5 to $1.5 \mathrm{~W} \cdot \mathrm{cm}^{-2}$ range. Additional substrate pre-heating from a heat source independently of the plasma discharge was used over the temperature range of 100 to $300{ }^{\circ} \mathrm{C}$, and elevated temperatures were then maintained throughout the plasma treatment. The duration of the plasma treatment was $15 \mathrm{~min}$. Details of the plasma reactor and the processing parameters used are published elsewhere [13].

EL was powered using the constant current (CC) regime with the positive terminal being normally connected to the ITO electrode. Reversed polarity powering (i.e. applying a negative voltage to the ITO electrode) was alternatively employed. EL measurements were performed as a function of EL emission wavelength. Additionally, the dependence of the EL intensity at $\lambda=390 \mathrm{~nm}$ on both the applied electric power and the time of electron injection was studied. The values of the voltage $V_{\mathrm{CC}}$ applied across the MOSLED structure during EL measurements were also recorded.

\section{Results and discussion}

Fig. 1 is a composite of EL spectra obtained from MOSLEDs that had been plasma treated at different plasma power densities. The EL spectra were recorded after the application of either positive or negative potential to the ITO electrode. Three features can be noticed. The EL spectra all show a peak at $390 \mathrm{~nm}$ and a shoulder at about $420 \mathrm{~nm}$. PT at the optimum power density of $0.7 \mathrm{~W} \cdot \mathrm{cm}^{-2}$ results in a slight increase in the EL intensity and broadening the spectrum compared to that for the untreated structure. Higher plasma power densities lead to a rapid decrease in the EL intensity. It is worth to point out that lower plasma power densities do not have any discernible effect on the EL spectra (not shown). Also, it should be noted that the PT itself was found to cause no changes in the Ge implant profile over the entire range of temperatures and powers used as confirmed by the Rutherford backscattering spectroscopy analysis (not shown).

Fig. 2 shows the EL intensity measured at $390 \mathrm{~nm}$ as a function of electric power applied to the device using the same two modes of electron injection as above, and for different plasma power densities. It is evident that the PT performed at a power density of $0.7 \mathrm{~W} \cdot \mathrm{cm}^{-2}$ leads to a considerable increase in the applied electric power compared to the untreated structure. Increasing the plasma power density to $1.5 \mathrm{~W} \cdot \mathrm{cm}^{-2}$ results in a reduction of the maximal applied electric power, which the device can still withstand.

Another important benefit of the plasma treatment is that it enables one to achieve an appreciable increase in the total injected electron charge just before the breakdown or the so-called charge to breakdown $Q_{\mathrm{BD}}$. 


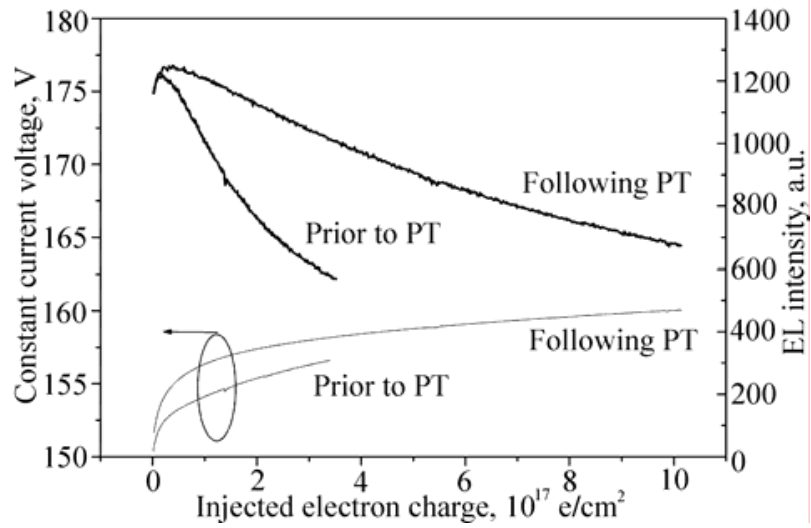

Fig. 3. EL intensity and constant current voltage as a function of electron injected charge prior to and following PT. The current density used was $2 \cdot 10^{-4} \mathrm{~A} \cdot \mathrm{cm}^{-2}$.

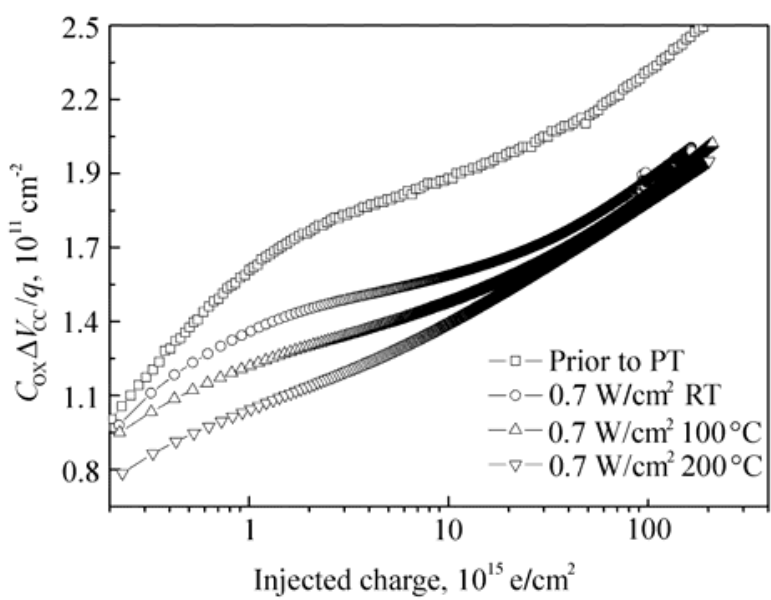

Fig. 4. Variation of the negative trapped charge with total injected charge prior to and following PT. The current density used was $2 \cdot 10^{-5} \mathrm{~A} \cdot \mathrm{cm}^{-2}$.

This is shown in Fig. 3, where the EL intensity is plotted as a function of injected electron charge. The increase in the total injected electron charge results directly in an improvement of the device lifetime. A frequently used measure of the EL durability is the time to halfluminance or when the EL light output drops to $50 \%$ of the original light output. As can be seen, an untreated MOSLED shows relatively low durability in terms of time to half-luminance. In contrast, a plasma treated MOSLED exhibits substantially improved durability, which is by a factor of about four greater than that of the untreated structure. Furthermore, there is an overall increase in the total EL intensity for the plasma treated device whereby one and the same intensity is achieved for injected times (or injected charges) that are longer by a factor of about 3.5 as compared to the untreated device. And finally, the values of $V_{\mathrm{CC}}$ for the plasmatreated MOSLEDs also show an increase as compared to the untreated devices (see Fig. 3).
The mechanism via which a MOSLED operates is believed to be based upon impact excitation of specific defect-type luminescent centers by hot electrons moving in the conduction band of the oxide [4]. More specifically, the structural defects produced in the oxide network by the implantation process are of the so-called oxygendeficient centers (ODC) type, in one of which (ODC I) the oxygen atoms of the original $\equiv \mathrm{Si}-\mathrm{O}-\mathrm{Si} \equiv$ bonding configuration are removed allowing replacement of the $\mathrm{Si}$ atoms by implanted $\mathrm{Ge}$ atoms and formation of $\mathrm{Si}-\mathrm{Ge}$ or Ge-Ge bonds [14]. At sufficiently high implant doses, the formation of nanoscale clusters of excess Ge atoms occurs. These clusters assist both the injection and the transport of electrons that in turn are accelerated to excite EL [5]. By this model, the results shown in Figs 3 and 4 serve to establish that the PT itself works in such a way as to reduce the amount of those defect complexes and states that have detrimental effects on the EL properties of the MOSLEDs while maintaining unchanged the population of the defect-type centers that mediate excitation of EL. The increase of $V_{\mathrm{CC}}$ during the electron injection time is associated mainly with the negative charge trapping in the oxide $[7,8]$.

An additional evidence of the favorable effect of PT on the quality of the oxide layer in a MOSLED can be obtained by calculating the electron trap parameters both before and after the plasma treatment. As can be seen in Fig. 3, $V_{\text {CC }}$ does not saturate above an injected charge of $10^{17} \mathrm{e} / \mathrm{cm}^{2}$, which may be attributed to the generation of new electron traps during high-field electron injection $[15,16]$. In our case, this phenomenon may manifest itself due to the fact that the oxide has been subjected to high dose Ge implantation (6 at. \%). Fig. 4 shows that, for injected charge values in excess of $10^{17} \mathrm{e} / \mathrm{cm}^{2}$, the plasma treatment using a power density of $0.7 \mathrm{~W} \cdot \mathrm{cm}^{-2}$ leads to a decrease in the trapping efficiency of the newly generated traps $\left(\eta=d Q_{t} / d Q_{\text {inj }}\right)$, where $Q_{t}=C_{\mathrm{OX}} \Delta V_{\mathrm{CC}}$. A computer fitting of the trapped charge in the oxide versus injected charge assuming the firstorder kinetics up to an injected charge of $10^{17} \mathrm{e} / \mathrm{cm}^{2}$, and trap generation for injected charge values above $10^{17} \mathrm{e} / \mathrm{cm}^{2}$ allows one to calculate the capture crosssection of the traps capturing the negative charge [17], and the effective trap generation probability (without extracting the occupation probability) [15]. For the particular implant conditions used, it yields the following capture cross-sections for these traps: $\sigma_{1}=$ $=1.6 \cdot 10^{-15} \mathrm{~cm}^{-2}, \sigma_{2}=1.2 \cdot 10^{-16} \mathrm{~cm}^{-2}, \sigma_{3}=7.8 \cdot 10^{-18} \mathrm{~cm}^{-2}$ and $\eta=4.6 \cdot 10^{-7}$. After PT at a plasma power density of $0.7 \mathrm{~W} \cdot \mathrm{cm}^{-2}$ and additional $200{ }^{\circ} \mathrm{C}$ heating there is a measurable reduction in both the cross-sections of the above-mentioned traps and the effective trap generation probability, namely $\sigma_{1}=1.1 \cdot 10^{-15} \mathrm{~cm}^{-2}, \quad \sigma_{2}=$ $=8.8 \cdot 10^{-17} \mathrm{~cm}^{-2}, \quad \sigma_{3}=6.5 \cdot 10^{-18} \mathrm{~cm}^{-2}$ and $\eta=3.1 \cdot 10^{-7}$, which serves as an evidence of the reduced electron capture in the dielectric. In addition, for the plasma treated MOSLEDs the voltage necessary to maintain the same CC is higher than that for the untreated devices 
(see Fig. 3). This may be explained in terms of the partial removal of those traps that influence adversely the process of charge transfer through the oxide. The observed reduction in the capture cross-sections for the negative charge traps after plasma processing while keeping the EL intensity much the same suggests a different nature of the traps responsible for the light emission and the attendant oxide degradation. At the same time, the reduction of the electron capture in the newly generated traps after PT might lead to an increase of the device lifetime. All these changes are believed to be associated with a relief of the internal mechanical stress in the oxide resulting from structural rearrangements.

The decrease in the EL intensity with increasing the plasma power density above $0.7 \mathrm{~W} \cdot \mathrm{cm}^{-2}$ (see Fig. 2) is most likely due to the annealing of the respective EL centers. It should be noted that the relative stability of the EL center that gives rise to the $420 \mathrm{~nm}$ violet emission compared to the EL center that produces the $380 \mathrm{~nm}$ violet emission suggests a larger capture crosssection of the latter. Indeed, the low-temperature RF plasma annealing of defects in silicon dioxide has been explained by the model of recombination enhanced defect reactions $[18,19]$ which takes into account electron and hole injection processes into the oxide during plasma treatment and energy extraction during the effective electron-hole recombination at the defects.

The use of light-mass chemically reactive species such as hydrogen and nitrogen is beneficial in two ways. First, the light-mass particles present in the plasma will not erode/etch the surface so aggressively as will do heavier-mass species like argon. Second, hydrogen is helpful in saturating the dangling bonds as well as in passivating and modifying various defect complexes [20], whereas nitrogen causes partial nitridation of the oxide surface, thus improving its structural quality. In effect, these two elements in combination provide favorable conditions for surface and defect modifications.

\section{Conclusions}

This work has demonstrated the beneficial effects of an optimized plasma treatment on the overall quality of Geimplanted MOSLEDs. These effects can be summarized as follows. First, such a treatment enables one to achieve an almost four-fold increase in the total injected electron charge just before breakdown $Q_{\mathrm{BD}}$, which in turn results in an improvement of the device lifetime. Second, treating MOSLEDs in a plasma discharge containing chemically reactive species like nitrogen and hydrogen leads to the appreciable restoration of the oxide matrix. Third, the plasma treatment causes a reduction in the capture cross section of some traps that are responsible for the accumulation of detrimental charges in the oxide, while maintaining unchanged the number of those defect complexes that give rise to the violet EL. Ultimately, the observed improvement of the MOSLEDs' quality is due to the partial removal of specific charge traps, defect passivation, recovery of the oxide network resulting from a relief of internal mechanical stresses, and bond rearrangement.

\section{Acknowledgments}

This work was supported by the German Bundesministerium für Bilding und Forschung (BMBF) under contract N WTR UKR 01/54. The authors are grateful to Dr. M. Voelskow for the RBS measurements.

\section{References}

1. K.V. Shcheglov, C.M. Yang, K.J. Vahula, and H.A. Atwater, Electroluminescence and photoluminescence of Ge-implanted $\mathrm{Si} / \mathrm{SiO}_{2} / \mathrm{Si}$ structures // Appl. Phys. Lett. 66 (6), p. $745-747$ (1995).

2. J.-Y. Zhang, X.-L. Wee, and X.-M. Bao, Electroluminescence and photoluminescence of $\mathrm{Ge}$ ${ }^{+}$-implanted $\mathrm{SiO}_{2}$ films thermally grown on crystalline silicon // Ibid. 71 (17), p. 2505-2507 (1997).

3. P. Knapek, B. Rezek, D. Muller, J.J. Grob, R. Levy, K. Luterova, J. Kocka, and I. Pelant, Blue electroluminescence from an $\mathrm{SiO}_{2}$ film highly implanted with $\mathrm{Si}^{+}$ions // Phys. status solidi (a) 167 (1), p. R5-R6 (1998).

4. L. Rebohle, J. von Borany, and W. Skorupa, Blue photo- and electroluminescence of silicon dioxide layers ion-implanted with group IV elements // Appl. Phys. B70, p. 1-21 (2000).

5. L. Rebohle J. von Borany, R. Borchert, H. Fröb, T. Gebel, M. Helm, W. Möller, and W. Skorupa, Efficient blue light emission from silicon the first integrated Si-based optocoupler // Electrochem. Solid State Lett. 4 (7), p. G57-G60 (2001).

6. M.V. Fischetti, Generation of positive charge in silicon dioxide during avalanche and tunnel electron injection // J. Appl. Phys. 57 (8), p. 2860-2879 (1985).

7. T. Gebel, L. Rebohle, W. Skorupa, A.N. Nazarov, I.N. Osiyuk, and V.S. Lysenko, Charge trapping in light-emitting $\mathrm{SiO}_{2}$ layers implanted with $\mathrm{Ge}^{+}$ions // Appl. Phys. Lett., 81 (14), p. 2575-2577 (2002).

8. A.N. Nazarov, I.N. Osiyuk, V.S. Lysenko, T. Gebel, L. Rebohle, and W. Skorupa, Charge trapping and degradation in $\mathrm{Ge}+$ ion implanted $\mathrm{SiO}_{2}$ layers during high-field electron injection // Microelectronics Reliability 42 (9-11), p. 1461-1464 (2002).

9. V.S. Lysenko, M.M. Lokshin, A.N. Nazarov, and T.E. Rudenko, RF plasma annealing of implanted MIS structures // Phys. status solidi (a) 88 (2), p. 705-712 (1985).

10. V.V. Artamonov, V.S. Lysenko, A.N. Nazarov, V.V. Strelchuk, M.Ya. Valakh, and I.M. Zaritskii, Relaxation of amorphous structure of implanted Si under RF plasma treatment: Raman and EPR study // Semicond. Sci. Technol. 6 (2), p. 1-5 (1990). 
11. A.N. Nazarov, V.I. Kilchitska, I.P. Barchuk, A.S. Tkachenko, and S. Ashok, Radio frequency plasma annealing of positive charge generated by Fowler-Nordheim electron injection in buried oxides in silicon // J. Vac. Sci. Technol. 18 (3), p. 1254-1261 (2000).

12. T. Gebel, Nanocluster-rich $\mathrm{SiO}_{2}$ layers produced by ion beam synthesis: electrical and optoelectronic properties, PhD Thesis, Dresden, FZ Rossendorf (2002).

13. A.N. Nazarov and V.S. Lysenko, RF plasma treatment as a method of radiation and thermal hydrogenation of Si microelectronics structures // Mikroelektronika 23 (4), p. 45-64 (1994) (in Russian).

14. L. Skuja, in: Defects in $\mathrm{SiO}_{2}$ and Related Dielectrics: Science and Technology, edited by G. Pacchioni et al. Kluwer, Dordrecht, Ser. II 2, p. 73 (2000).

15. Y. Nissan-Cohen, J. Shapir, and D. FrohmanBentchkowsky, Trap generation and occupation dynamics in $\mathrm{SiO}_{2}$ under charge injection stress // J. Appl. Phys. 60 (6), p. 2024-2035 (1986).
16. J.F. Zhang, S. Taylor, and W. Eccleston, Electron trap generation in thermally grown $\mathrm{SiO}_{2}$ under Fowler-Nordheim stress // Ibid. 71 (2), p. 725-734 (1992).

17. A.N. Nazarov, T. Gebel, L. Rebohle, W. Skorupa, I.N. Osiyuk, and V.S. Lysenko, Trapping of negative and positive charge in $\mathrm{Ge}^{+}$ion implanted silicon dioxide layers subjected to high-field injection // Ibid. 94 (7), p. 4440-4448 (2003).

18. J.C. Kimmerling, Recombination enhanced defect reactions // Solid State Electron. 21 (11-12), p. 1391-1401 (1978).

19. T.P. Ma, M.P. Chin, RF annealing mechanisms in metal-oxide semiconductor structures - an experimental simulation // J. Appl. Phys. 51 (10), p. 54585463 (1980).

20. A.N. Nazarov, V.M. Pinchuk, V.S. Lysenko, T.V. Yanchuk, and S. Ashok, Enhanced activation of implanted dopant impurity in hydrogenated crystalline silicon // Phys. Rev. B58 (7), p. 35223526 (1998). 